University of Wollongong

Research Online

Australian Institute for Innovative Materials -

Papers

Australian Institute for Innovative Materials

$1-1-2018$

Feasibility and Characterization of Common and Exotic Filaments for Use in 3D Printed Terahertz Devices

Andrew Squires

University of Wollongong, ads786@uowmail.edu.au

R A. Lewis

University of Wollongong, roger@uow.edu.au

Follow this and additional works at: https://ro.uow.edu.au/aiimpapers

Part of the Engineering Commons, and the Physical Sciences and Mathematics Commons

Research Online is the open access institutional repository for the University of Wollongong. For further information contact the UOW Library: research-pubs@uow.edu.au 


\title{
Feasibility and Characterization of Common and Exotic Filaments for Use in 3D Printed Terahertz Devices
}

\author{
Abstract \\ Recent years have seen an influx of applications utilizing 3D printed devices in the terahertz regime. The \\ simplest, and perhaps most versatile, modality allowing this is Fused Deposition Modelling. In this work, a \\ holistic analysis of the terahertz optical, mechanical and printing properties of 17 common and exotic 3D \\ printer filaments used in Fused Deposition Modelling is performed. High impact polystyrene is found to be \\ the best filament, with a useable frequency range of $0.1-1.3 \mathrm{THz}$, while remaining easily printed. Nylon, \\ polylactic acid and polyvinyl alcohol give the least desirable terahertz response, satisfactory only below \\ $0.5 \mathrm{THz}$. Interestingly, most modified filaments aimed at increasing mechanical properties and ease of \\ printing do so without compromising the useable terahertz optical window. \\ Disciplines \\ Engineering | Physical Sciences and Mathematics

\section{Publication Details} \\ Squires, A. D. \& Lewis, R. A. (2018). Feasibility and Characterization of Common and Exotic Filaments for \\ Use in 3D Printed Terahertz Devices. Journal of Infrared, Millimeter, and Terahertz Waves, 39 (7), 614-635.
}




\title{
Feasibility and Characterization of Common and Exotic Filaments for Use in 3D Printed Terahertz Devices
}

\author{
A. D. Squires ${ }^{1}$ (D) R. A. Lewis ${ }^{1}$
}

Received: 11 November 2017 / Accepted: 11 May 2018/

Published online: 5 June 2018

(C) Springer Science+Business Media, LLC, part of Springer Nature 2018

\begin{abstract}
Recent years have seen an influx of applications utilizing 3D printed devices in the terahertz regime. The simplest, and perhaps most versatile, modality allowing this is Fused Deposition Modelling. In this work, a holistic analysis of the terahertz optical, mechanical and printing properties of 17 common and exotic $3 \mathrm{D}$ printer filaments used in Fused Deposition Modelling is performed. High impact polystyrene is found to be the best filament, with a useable frequency range of $0.1-$ $1.3 \mathrm{THz}$, while remaining easily printed. Nylon, polylactic acid and polyvinyl alcohol give the least desirable terahertz response, satisfactory only below $0.5 \mathrm{THz}$. Interestingly, most modified filaments aimed at increasing mechanical properties and ease of printing do so without compromising the useable terahertz optical window.
\end{abstract}

Keywords Terahertz $\cdot \mathrm{THz} \cdot 3 \mathrm{D}$ printing $\cdot$ Additive fabrication $\cdot \mathrm{FDM}$

\section{Introduction}

Recent years have seen additive manufacturing (3D printing) revolutionize many aspects of the manufacturing industry, allowing cheap, rapid and customizable fabrication of elements to be used functionally or in prototyping. In 3D printing, items are generated via additive methods, which sees final products built up a single layer

A. D. Squires

ads786@uowmail.edu.au

1 Institute for Superconducting and Electronic Materials and School of Physics, University of Wollongong, Wollongong, NSW 2522, Australia 
at a time from the ground up. This is in direct contrast to other subtractive methods, where material is removed from bulk via, for example, milling on a CNC machine or turning on a lathe.

The most common and cost-efficient form of 3D printing is Fused Deposition Modelling (FDM), developed by Stratasys [1]. In FDM, a thermoplastic polymer is extruded onto a print bed, following the generated paths in a sliced model's G-code. The polymer material is pulled from a spool by a drive gear into a hot end. This consists of a heater block in thermodynamic equilibrium with the extruder nozzle. The plastic is melted in this section with pressure from the drive gear ensuring material is extruded onto the print bed. In some cases, this print bed may be heated. The temperatures of the hot end are generally in the order of $200^{\circ} \mathrm{C}$, with print bed temperatures ranging from room temperature to $130^{\circ} \mathrm{C}$. The exact temperatures needed depend on the polymer thermal properties, specifically the glass transition temperature and coefficient of thermal expansion in conjunction to the hardware of the $3 \mathrm{D}$ printer setup. The most common plastics used with this technology are acrylonitrile butadiene styrene (ABS) and polylactic acid (PLA) [1]. These provide the most cost-effective and easy-to-use form of 3D printing [2]. Recently, engineered variants of these polymers have become available, as well as many other exotic filaments.

$\mathrm{THz}$ methods stand to benefit substantially from this technology. Typical $\mathrm{THz}$ optics consist of diffraction gratings, lenses [3], waveguides/ dielectric fibers [4] and prisms [5]. Conventionally, optical elements have been made by subtractive methods, such as machining lenses on a lathe [6], or cutting grooves in a substrate to form a diffraction grating [7, 8].

\section{Summary of 3D Printing and Terahertz Research}

3D printed THz devices have rapidly developed over recent years [9]. This is especially apparent in passive dielectric devices. Diffraction gratings [10] have been demonstrated, as have a range of lenses [11-13] and Quasi-Wollaston prisms [27]. In these devices, bulk absorption in the thermoplastic used leads to high absorption losses. Hyperbolic fresnel lenses tailor-made for THz linear scanners [14] have been developed, emphasizing the advantages of rapid, customizable prototyping evident in $3 \mathrm{D}$ printing $\mathrm{THz}$ optics. A range of 3D printed waveguides [15] and antennas [16] have emerged including plasmonic guides for surface plasma polariton propagation [17] as well as electromagnetic crystal waveguides with periodic triangular structures surrounding an air core [18]. Similarly, hyperuniform waveguides with periodic disordered reflectors surrounding an air core [19] have produced measured photonic band gaps. Further examples of hollow core waveguides with periodic cladding structure are given in Ref. [20]. A 3D printed anti-reflection waveguide structure has shown low loss, close to zero dispersion of $\mathrm{THz}$ waves [21]. Interestingly, arising from research pertaining to $\mathrm{THz}$ communications is a $3 \mathrm{D}$ printed leaky waveguide antenna for $\mathrm{THz}$ frequency division multiplexing [22]. In a slightly more exotic case, guide cladding is customized by 3D printing to contain metal 
inclusions inserted post-production [23]. Rapid prototyping has proved fruitful in testing periodic designs for use in a two-dimensional periodic surface lattice required in electron beam guidance [24]. Further complicated devices have been realized including dielectric metamaterials [25]. With the advent of metallicized 3D printer filament, a recent work has even developed 3D printed metallic rectangular waveguides [26]. A summary of 3D printed elements designed for $\mathrm{THz}$ frequencies is given in Table 1. As can be seen, most devices are generated via high-end stereolithography (SLA) and polyjet (PJT) methods using a form of proprietary photocurable resin. This is generally due to superior resolutions available via these methods. However, with a large capital investment, considerable running costs and minimal compatibility of non-manufacturer resins/polymers, these methods are not desirable for many $\mathrm{THz}$ labs. Thus, fully understanding and identifying usable alternatives in the cheap and versatile FDM printers becomes desirable. This is further emphasized with many proprietary materials containing in-house modifications, which in some cases alter the $\mathrm{THz}$ response, despite remaining unchanged to visible light. These modifications are aimed at enhancing optical (visible), mechanical and printing properties of the thermoplastic material.

Table 1 Summary of 3D printed $\mathrm{THz}$ devices

\begin{tabular}{lll}
\hline Printed device & Material used & References \\
\hline Diffractive/ flat optics & PLA & Squires [10] \\
& TOPAS & Busch [13] \\
Nylon & Suszek [14] \\
Lenses & TOPAS & Busch [13] \\
& Nylon & Furlan [11] \\
& Visijet Crystal & Squires [10] \\
& FullCure835 VeroWhite & Zhang [12] \\
& Polystyrene & Busch [50] \\
Not provided & Yi [28] \\
Beamplitters/prisms & Modified ABS (Bendlay) & Hernandez-Serrano [27] \\
Waveguides and antennas & Not provided & Karl [22] \\
& Multiple photocurable resins & Otter [20] \\
& Visijet Crystal & Ma [19], Yudasari [23, 31] \\
& & Vogt [21, 32] \\
& Photocurable resin & Wu [18], Pandey [17] \\
PlasClear and Asiga & Li [29] \\
Periodic structures & Polystyrene & Weidenbach [15] \\
& Photocurable resin & Gurvitz [25], Zhou [30], \\
& & Phipps [24] \\
& Cu-15 Sn powder & Zhang [26] \\
& & Headland [33] \\
\hline
\end{tabular}




\section{Experiment}

Experiments were performed on a Makerbot Replicator 2X: Experimental 3D printer, Fig. 1. (Two other 3D printers we have used are also shown in Fig. 1.) This printer was chosen specifically for this task for the following reasons. Firstly, the printer had to be placed at a price point easily accessible for research labs while maintaining the core advantages of 3D printing in the THz: cost-effectiveness and customization. The Replicator $2 \mathrm{X}$ is designed specifically with this in mind. The printer contains all of the hardware required to print the full range of filaments, while allowing high levels of user input to easily modify, exchange and upgrade working parts as required for printing with exotic filaments. This is coupled with high levels of access to the printer's source code, permitting adjustments to all possible print parameters and thus ideal for printing with non-standard filaments. Most inexpensive 3D printers are designed specifically for one type of filament, with print parameters optimized accordingly, without access for manipulation. Typically, these printer manufacturers seal many of the working parts such that modifying them to print non-proprietary filaments results in a void of warranty and high risk of serious damage.

Seventeen common and exotic filaments have been investigated including the following: polylactic acid (PLA), acrylonitrile butadiene styrene (ABS), nylon, polycarbonate (PC), polypropylene (PP), polyethylene terephthalate (PET), high impact polystyrene (HIPS) and polyvinyl alcohol (PVA) with their corresponding modifications and blends. Where possible, pure filament was obtained free from colorizing agents and dyes.

The optimal build parameters for each filament were found experimentally from trial and error using manufacturer recommendations as a starting point. These are summarized in Tables 2 and 3. In the cases, where a range of successful extrusion temperatures were present, samples were printed at the highest possible temperature. This enables better merging between adjacent strips of thermoplastic, thus higher homogeneity of the plastic throughout the sample, reducing scattering of the $\mathrm{THz}$ waves from individual strands of material.

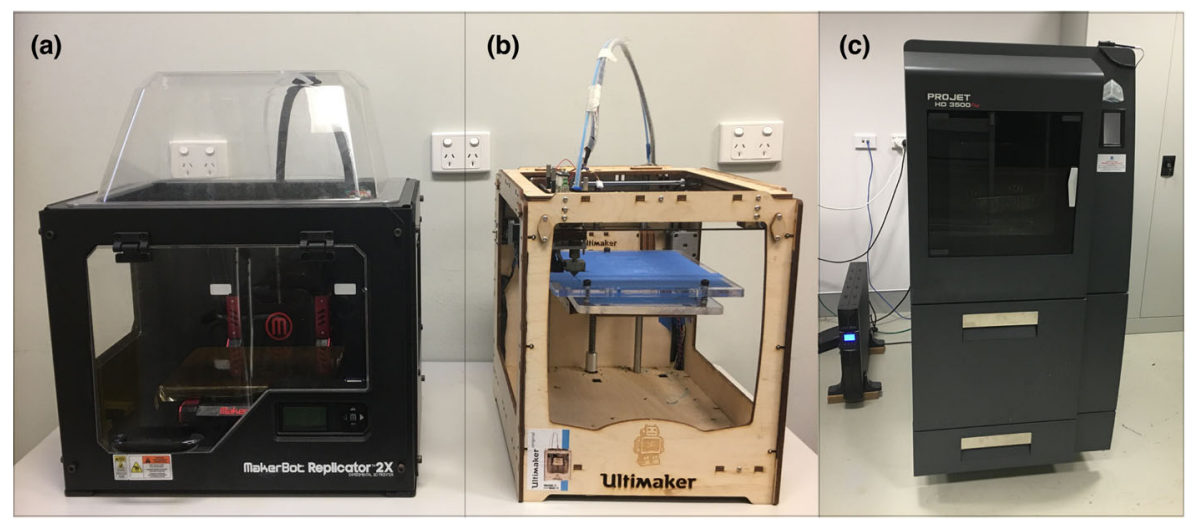

Fig. $13 D$ printers available at UOW. a Makerbot Replicator 2X: Experimental 3D printer. b Ultimaker 1: 3D printer. c 3D Systems Projet HD3500 Plus MJT 3D Printer 
Table 2 Optimized filament extrusion and build plate temperatures

\begin{tabular}{|c|c|c|c|}
\hline Filament & Modification & Extrusion temperature $\left({ }^{\circ} \mathrm{C}\right)$ & Build plate temperature $\left({ }^{\circ} \mathrm{C}\right)$ \\
\hline \multirow[t]{2}{*}{ PLA } & Standard & $180^{\circ} 210^{\circ} 240^{\circ} 270^{\circ} 300^{\circ}$ & Not needed \\
\hline & PLA/PHA blend & ${ }^{\circ} 180^{\circ} 210^{\circ} 240^{\circ} 270^{\circ} 300^{\circ}$ & Not needed \\
\hline \multirow[t]{2}{*}{ ABS } & Standard & $180^{\circ} 210^{\circ} 240^{\circ} 270^{\circ} 300^{\circ}$ & 110 \\
\hline & Conductive ABS & $180^{\circ} 210^{\circ} 240^{\circ} 270^{\circ} 300^{\circ}$ & 110 \\
\hline \multirow[t]{5}{*}{ Nylon } & Standard (white) & $0^{\circ} 180^{\circ} 210^{\circ} 240^{\circ} 270^{\circ} 300^{\circ}$ & $70-100$ \\
\hline & 645 & $180^{\circ} 210^{\circ} 240^{\circ} 270^{\circ} 300^{\circ}$ & $70-100$ \\
\hline & 910 & $180^{\circ} 210^{\circ} 240^{\circ} 270^{\circ} 300^{\circ}$ & $70-100$ \\
\hline & Bridge & $0^{\circ} 180^{\circ} 210^{\circ} 240^{\circ} 270^{\circ} 300^{\circ}$ & $70-100$ \\
\hline & PCTPE & $180^{\circ} 210^{\circ} 240^{\circ} 270^{\circ} 300^{\circ}$ & 40 \\
\hline \multirow[t]{2}{*}{$\mathrm{PC}$} & Standard & $180^{\circ} 210^{\circ} 240^{\circ} 270^{\circ} 300^{\circ}$ & $>120$ \\
\hline & PC/ABS blend & $180^{\circ} 210^{\circ} 240^{\circ} 270^{\circ} 300^{\circ}$ & $>120$ \\
\hline PP & Standard & $180^{\circ} 210^{\circ} 240^{\circ} 270^{\circ} 300^{\circ}$ & $>120$ \\
\hline \multirow[t]{3}{*}{ PET } & T-Glase & ${ }^{\circ} 180^{\circ} 210^{\circ} 240^{\circ} 270^{\circ} 300^{\circ}$ & $<68$ \\
\hline & Colorfabb-XT & $0^{\circ} 180^{\circ} 210^{\circ} 240^{\circ} 270^{\circ} 300^{\circ}$ & $60-70$ \\
\hline & PETG & ${ }^{\circ} 180^{\circ} 210^{\circ} 240^{\circ} 270^{\circ} 300^{\circ}$ & Not needed \\
\hline HIPS & Standard & $180^{\circ} 210^{\circ} 240^{\circ} 270^{\circ} 300^{\circ}$ & 110 \\
\hline PVA & Standard & $150^{\circ} 180^{\circ} 210^{\circ} 240^{\circ} 270^{\circ} 300^{\circ}$ & Not needed \\
\hline
\end{tabular}


Table 3 Limits of filament layer height and print speed

\begin{tabular}{llll}
\hline Filament & Modification & Min layer height $(\mathrm{mm})$ & Print speed $(\mathrm{mm} / \mathrm{s})$ \\
\hline PLA & Standard & 0.1 & 90 \\
& PLA/PHA blend & 0.1 & 90 \\
ABS & Standard & 0.05 & 90 \\
& Conductive ABS & 0.2 & 30 \\
Nylon & Standard (white) & 0.1 & 50 \\
& 645 & 0.1 & 70 \\
& 910 & 0.1 & 90 \\
& Bridge & 0.1 & 70 \\
& PCTPE & 0.1 & 70 \\
PC & Standard & 0.1 & 45 \\
& PC/ABS blend & 0.1 & 45 \\
PP & Standard & 0.1 & 30 \\
PET & T-Glase & 0.1 & 60 \\
& Colorfabb-XT & 0.1 & 60 \\
& PETG & 0.1 & 60 \\
HIPS & Standard 110 & 0.1 & 90 \\
PVA & Standard & 0.1 & 10 \\
\hline
\end{tabular}

Samples for spectroscopic purposes were designed as $50 \mathrm{~mm} \times 50 \mathrm{~mm} \times 1 \mathrm{~mm}$ squares. The geometry was found to be optimal for mounting in THz-TDS equipment, while providing suitable transmitted signal for characterization measurements across a wide frequency range.

Transmission spectra were taken using an Advantest TAS7400TS time domain spectrometer. The experimental apparatus was purged with dry air. Two sensors inside the system ensured the humidity was below $0.1 \%$ before commencing measurements. A preset spectral resolution of $15 \mathrm{GHz}$ was chosen. 16384 rapid scans were averaged to produce the final $\mathrm{THz}$ spectrum.

\section{Results}

\subsection{Polylactic Acid}

PLA is one of the most commonly used thermoplastics in 3D printing. The main reasons for this are twofold: Firstly, PLA is eco-friendly, being biodegradable and derived from natural products such as corn, sugar cane and potatoes [34]. Thus, PLA is seen as a sustainable alternative to petroleum-based polymers across many applications [35]. Furthermore, PLA uses 25-55\% less energy in production than its petroleum-derived counterparts [36]. Secondly, PLA's thermal properties are well suited to $3 \mathrm{D}$ printing. A relatively low glass transition temperature of $60^{\circ} \mathrm{C}$ and melting point of $153^{\circ} \mathrm{C}$ [37] allows printer extrusion temperatures of around $190^{\circ} \mathrm{C}$, easily accessible by all commercial 3D printers. Additionally, a relatively low 
thermal expansion coefficient of $70 \times 10^{-6}{ }^{\circ} \mathrm{C}^{-1}$ [38] gives negligible warping of printed models and circumvents the need of a heated print bed. Consequently, PLA is the easiest and most popular filament to print with. In terms of THz printing, PLA's toughness is its only downside. PLA prints will be very brittle, consequently limiting applications where high stress is present [36]. This will pose problems for prints in a laboratory context where, for example, a part may need to be fixed rigidly to an optical bench. However, since $\mathrm{THz} 3 \mathrm{D}$ printed devices are predominately optical in nature, this will generally not be an issue, especially considering the relative fragility of many current optical $\mathrm{THz}$ components such as beamsplitters.

To date, many approaches have been taken to improve PLA's mechanical properties, including various types of copolymerization and blending. The latter of these accounts for most efforts in improving PLA [36]. Poly(hydroxyalkanoate) (PHA), an aliphatic polyester produced by micro-organisms, has been found to drastically improve toughness upon blending with PLA. The PHA has the effect of lowering the melting point and overall crystallinity of the polymer structure, leading to an increase in toughness. One study [39] showed an increase in energy-to-break toughness of PLA when blended in a 90/10 ratio (per weight) with PHA from $\sim 0.25 \mathrm{Nm}$ to $\sim 2.0 \mathrm{Nm}$. This dropped to $\sim 1.4 \mathrm{Nm}$ with a $80 / 20$ ratio before, interestingly, reducing back to $<0.25 \mathrm{Nm}$ for PHA content above $40 \%$.

Figure $2 \mathrm{a}$ shows the absorption coefficient of PLA and PLA/PHA blended 3D printer filaments in the $\mathrm{THz}$ regime. The absorption curve is featureless, increasing strongly with frequency, as is common in this region for dielectric materials. The standard and blended PLA filaments have identical spectra in this region.

Figure $3 \mathrm{a}$ interestingly shows that despite the similar absorption, the refractive indices of the filaments differ. For PLA, the refractive index is $1.56 \pm 0.02$ across the spectrum. For PLA/PHA blended filament, this shifts to $1.64 \pm 0.02$. Both filaments appear to have a slight decrease in refractive index across the frequency range. This is consistent with the $\mathrm{THz}$ data shifting towards the visible refractive index of PLA calculated as 1.465 at $589 \mathrm{~nm}$ from the sodium D-line [40].

The transmissive properties of the blended filament are dominated by the PLA. No change in the absorption coefficient is present despite a mixture of 10-20\% PHA. It is only in the refractive index that the differing filament composition is revealed. Thus, for 3D printing of transmissive optical devices for $\mathrm{THz}$ applications, the filaments are identical. Considering the more desirable mechanical properties of the PLA/PHA blend, this filament becomes the better option for this task.

However, the overall use of these filaments in the THz regime is still limited to thin devices and low photon energies. From Fig. 2a, a bulk material of only $1 \mathrm{~cm}$ thickness results in over $99 \%$ tranmissive losses for frequencies above $0.8 \mathrm{THz}$. For any frequency above this, these filaments are not feasible for optical applications. Below $0.5 \mathrm{THz}$, with the absorption coefficient dropping below $10 \mathrm{~cm}^{-1}$, thin optical devices, such as diffraction gratings, are achievable. Lenses and other applications that require a bulk of material will still be largely ineffective in this region without more complicated design, for example, removing the bulk of a standard lens in a fresnel lens.

In terms of the printability, PLA and PLA/PHA are at the forefront. The addition of PHA has no appreciable effects in the ease of printing models when compared 


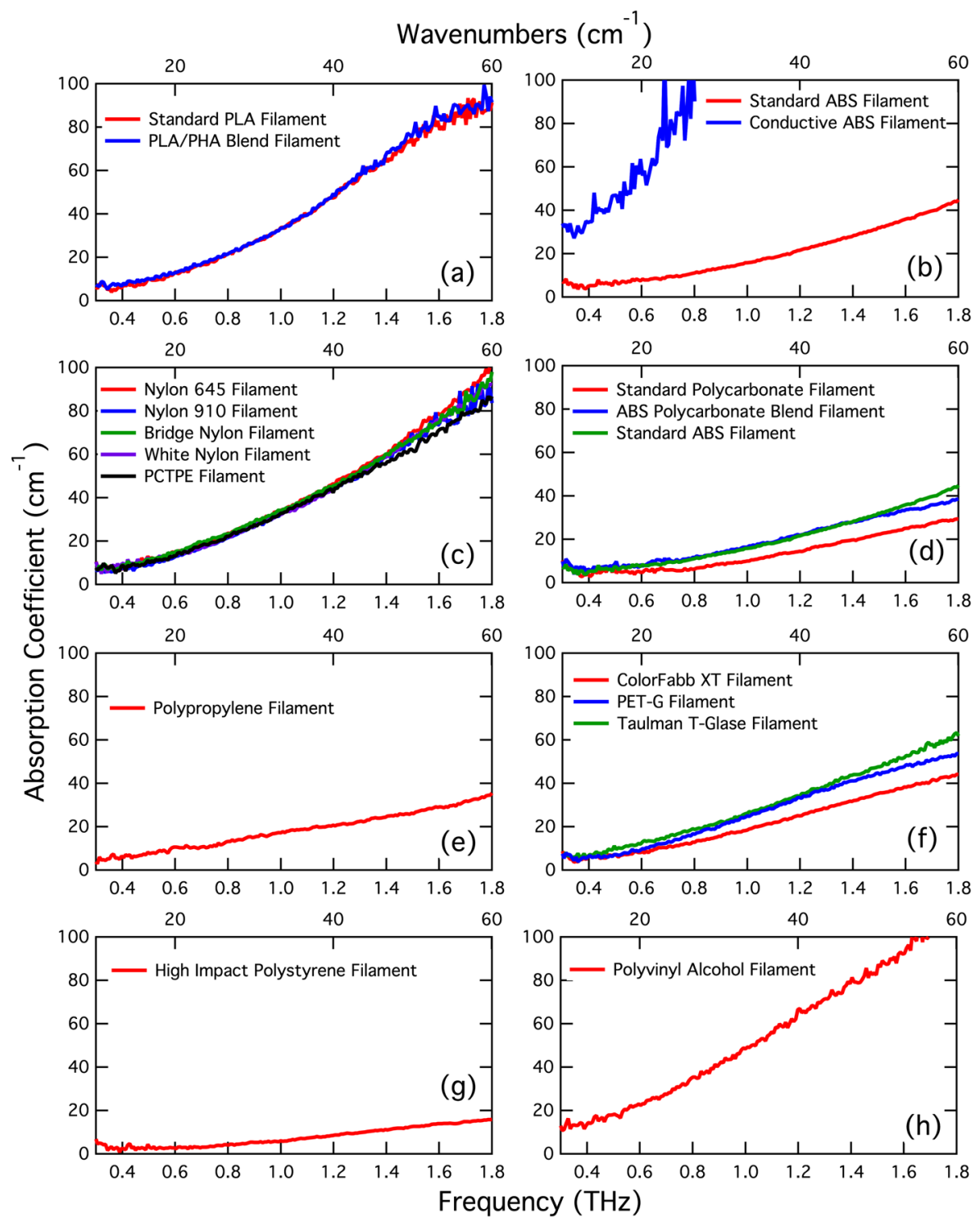

Fig. 2 Absorption coefficients of 3D printed plastics in the THz regime. Each panel corresponds to the following filament type; a PLA, b ABS, $\mathbf{c}$ nylon, $\mathbf{d}$ polycarbonate, $\mathbf{e}$ polypropylene, $\mathbf{f}$ PET, $\mathbf{g}$ polystyrene, h PVA

to standard PLA. Altering print parameters such as extrusion temperatures and feed rates was found to be unnecessary when changing between the two filaments. Overall, both filaments are easily printed. No heated bed is required due to low thermal expansion and models will stick to almost any build surface. Hence, printing accurate geometries, as is desirable for optical THz elements, is comparatively very easy. This 


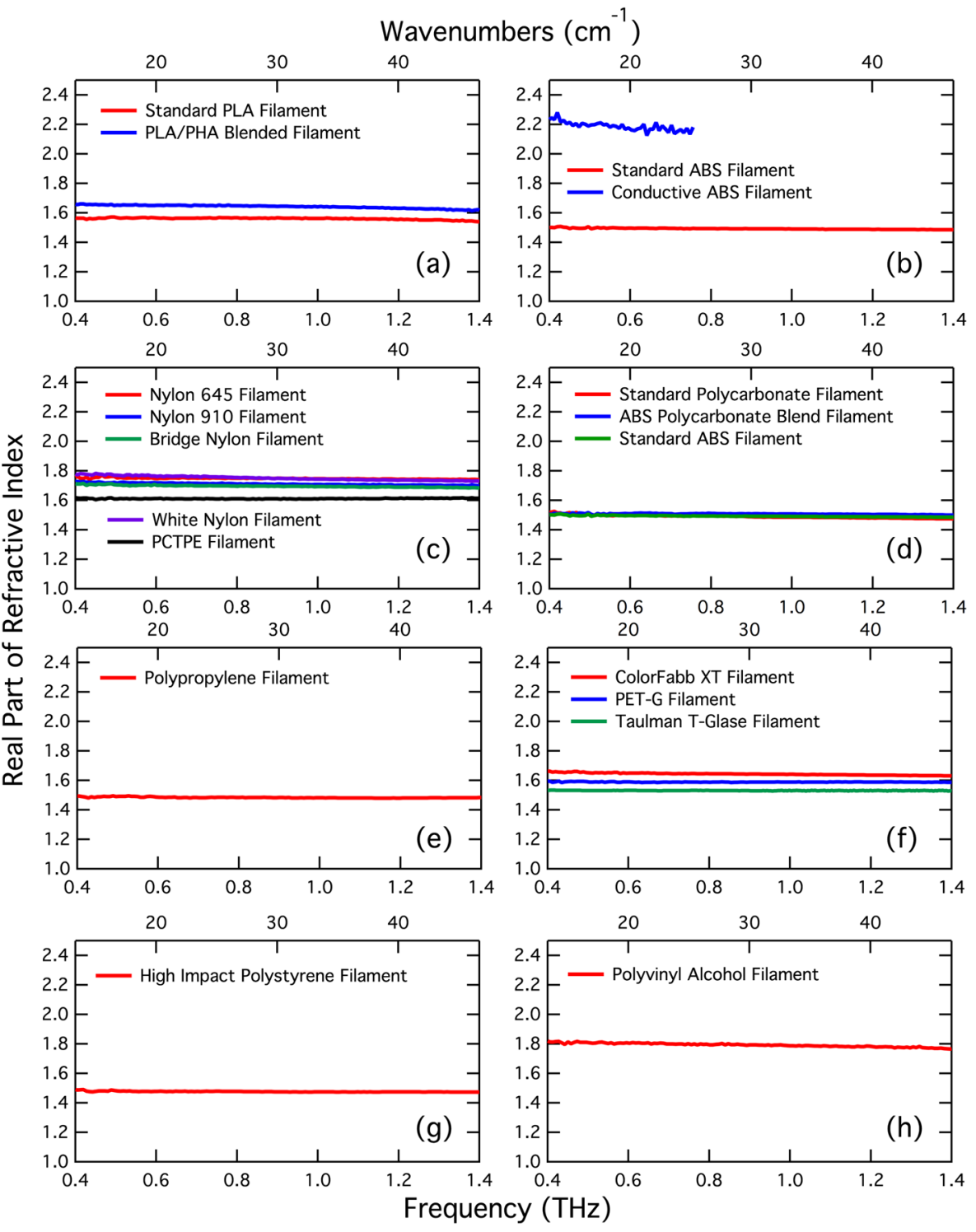

Fig. 3 Real part of refractive indices of 3D printed plastics in the $\mathrm{THz}$ regime. Each panel corresponds to the following filament type; a PLA, b ABS, c nylon, d polycarbonate, e polypropylene, $\mathbf{f}$ PET, g polystyrene, $\mathbf{h}$ PVA

is reflected in the use of PLA in almost all entry level 3D printers with novice users in mind. This is advantageous when considering that thin and precise components need to be printable for these filaments to be of use in $3 \mathrm{D}$ printing functional $\mathrm{THz}$ optics. 


\subsection{Acrylonitrile Butadiene Styrene}

Acrylonitrile butadiene styrene (ABS) is a very commonly used amorphous thermoplastic polymer. ABS consists of highly desirable mechanical properties, which drive its large use as a 3D printer filament. Alongside PLA, ABS is the most popular filament used in FDM 3D printers. ABS is a tough, stiff polymer that exhibits chemical, heat and moisture resistance [41]. In turn, 3D printed ABS parts extend beyond novel models and prototypes into structural components for equipment such as drones [42]. Despite the superior mechanical properties, ABS polymer is not biodegradable as is the case for many of its competitors. This has spearheaded research into deriving 3D printable polymers with the mechanical properties of $\mathrm{ABS}$, but the environmental properties of PLA [43].

Thermally, ABS has a desirable glass transition temperature of $112.6^{\circ} \mathrm{C}$ [44] leading to extrusion temperatures typically in the range of $230^{\circ} \mathrm{C}$, well within the capabilities of all FDM 3D printers. Unfortunately, a thermal expansion coefficient of $100 \times 10^{-6}{ }^{\circ} \mathrm{C}$ results in prints susceptible to warping. Consequently, printing in $\mathrm{ABS}$ requires the use of a heated print bed, in the vicinity of $110^{\circ} \mathrm{C}$ in conjunction to good bed adhesion to navigate this issue. Bed adhesion is improved by printing on polyimide (kapton) tape as well as preparing the build surface with glue, hairspray or a thin layer of acetone.

Although ABS polymers, like many plastics, have the desirable property of a high intrinsic electrical resistance, many applications would benefit from electrical conductivity. Accordingly, research has led to the development of polymer complexes with enhanced conductivity compared to their original composition. This is typically achieved through blending polymetric materials with a conductive filler, such as metal fibers and carbon [45]. This would be desirable for FDM printing, as such an experimental carbon-blended ABS has been developed, sold under the proprietary name 'Conductive ABS'.

Figure $2 \mathrm{~b}$ gives the absorption coefficients for ABS and conductive ABS filaments. Standard ABS shows a steady increase with photon energy with an absorption of $<10 \mathrm{~cm}^{-1}$ below $0.75 \mathrm{THz}$ rising to $>40 \mathrm{~cm}^{-1}$ above $1.7 \mathrm{THz}$. Besides this, the spectra is featureless for this filament. A significant increase in absorption is present for conductive ABS filament, as expected for conducting materials. The absorption coefficient remains above $30 \mathrm{~cm}^{-1}$ at low frequency, sharply reaching $>100 \mathrm{~cm}^{-1}$ beyond $0.8 \mathrm{THz}$. At this point, the data is cut-off as the highest absorption coefficient measurable by the system is reached. Furthermore, the small transmissive window corresponds to the lowest dynamic range available in the experimental setup, hence the low signal to noise for this sample.

In Fig. 3b, the refractive indices for standard and conductive ABS are $1.49 \pm 0.01$ and $2.19 \pm 0.08$, respectively. As expected, the conductive variant shows an appreciably higher refractive index, above the range typical for bulk dielectric materials. The refractive index for conductive filament shows a slight decrease with frequency, while standard ABS is steady across the THz range.

In terms of $3 \mathrm{D}$ printing optics for the $\mathrm{THz}$ region, standard $\mathrm{ABS}$ shows some promise. The absorption coefficient is $<10 \mathrm{~cm}^{-1}$ for frequencies below $0.75 \mathrm{THz}$ and approaches $5 \mathrm{~cm}^{-1}$ in the $0.4-\mathrm{THz}$ region. Thus, useable transmission is found in this region for the production of quasi-optics. 
Despite the claim, the overall conductivity of the conductive ABS filament is quite poor, given as $1 \times 10^{-4} \mathrm{~S} / \mathrm{m}$, somewhat in the 'mid-range' between plastics and conductors. This limits its use for $\mathrm{THz}$ devices. The conductivity is increased enough from standard ABS to effect the optical spectrum to the point of rendering these devices unfeasible, but not enough to open up applications in printed devices such as reflective optics and waveguides, typically engineered from metals. Figure 4 shows the reflectance of the conductive ABS to remain below 0.25 across the spectrum for a polished, flat surface. Despite this, the improvement in conductivity from standard ABS is encouraging.

ABS printability is generally fairly easy provided the $3 \mathrm{D}$ printer contains the required hardware. A heated print bed is the minimum requirement, with a closed print environment desirable to minimize warping issues. For most novice users, slight warping may not be an issue, but for printing functional $\mathrm{THz}$ optics, this will become a significant problem, especially if the structural changes with cooling are not uniform. This implies a careful print setup with detail taken in optimizing print parameters for each device is necessary. This requires more than a basic level of user expertise. Thus, successful prints with high geometric precision are feasible for the $\mathrm{THz}$ regime below $0.75 \mathrm{THz}$, but care must be taken in the print and design process.

Further issues arise for conductive ABS when printability is considered. Generated models are 'chalky' in appearance with smooth, accurate designs not feasible, with a limiting resolution of $2 \mathrm{~mm}$, observed experimentally. Smoothing models postproduction can improve this problem, but is not sufficient for designs with internal structures. Also, fine particles of carbon are released into the air during prints. These can be found deposited over the printer after some time, raising questions about the effects on the lifetime of moving parts. Hence, safety issues arise in breathing in these particulates, forcing prints to be performed in well-ventilated areas or fume hoods. Factoring the required resolution for conductive $\mathrm{THz}$ devices, like resonators and metamaterials, is well below those available with this filament, with the outlined

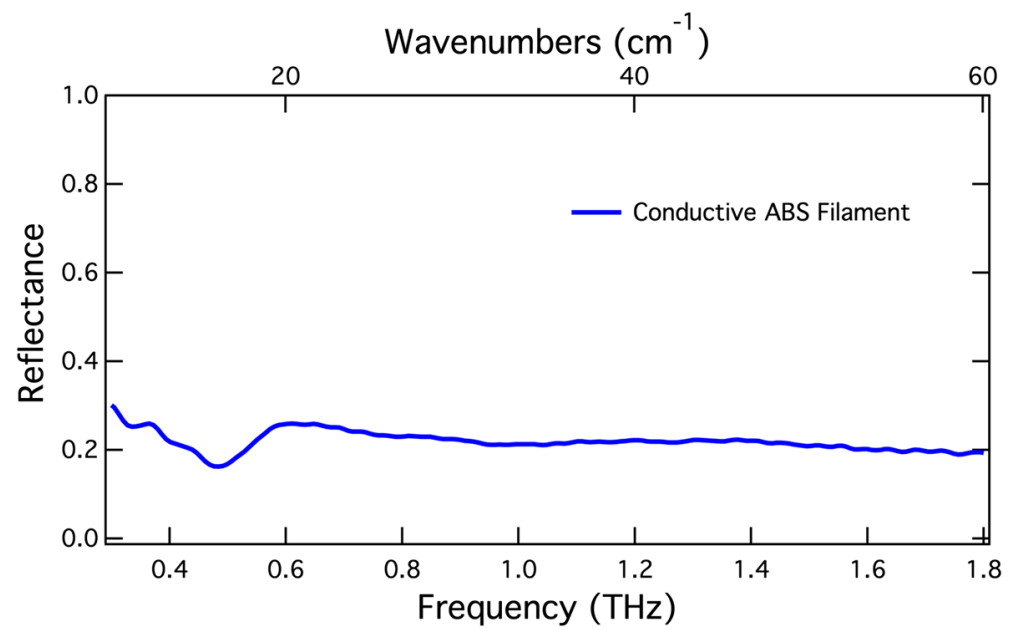

Fig. 4 Reflectance of conductive ABS filament. Data is presented as a ratio to an approximately perfect reflector (gold-plated mirror) 
safety concerns; it is concluded that conductive ABS shows poor prospects in $3 \mathrm{D}$ printing functional $\mathrm{THz}$ devices.

\subsection{Nylon}

Since the inception of FDM 3D printing technology, nylon has steadily become more accessible and functional as a filament. Standard nylon filaments are notoriously difficult to print with for many reasons. Firstly, relatively large thermal expansions ranging from $(8-10) \times 10^{-5}{ }^{\circ} \mathrm{C}^{-1}$ for polymers suitable for $3 \mathrm{D}$ printing result in large warping issues for printed parts. This is exaggerated by high print temperatures above $250^{\circ} \mathrm{C}$ required for successful nozzle extrusion. Secondly, layer adhesion and overall strength are poor due to the lack of compression provided by FDM printers. In industry, the strength and desirability of these nylon polymers are realized in the compression involved in the injection moulding process. This is simply not achievable with a filament 3D printer [46]. A more general issue with nylons that extends across industry is its large water absorption. For example, for standard nylon-6, a $2.7 \%$ equilibrium water absorption and $9.5 \%$ saturation level were observed at $50 \%$ relative humidity [47]. In response to these issues, a series of nylon filaments have been developed, each aiming to minimize one or all of these issues, while filling a certain niche in desirable mechanical properties. To investigate $\mathrm{THz}$ characteristics, plain white nylon filament was obtained as well as a series of engineered variants developed by taulman3D [46]:

- Nylon 645 - Produced to increase polymer bonding, reduce thermal expansion effects while maintaining high chemical resistance.

- Nylon 910 - Engineered to meet high performance needs. Minimizes thermal expansion beyond Nylon 645, gives a large range of chemical resistance while simultaneously consisting of a very high tensile strength.

- Bridge Nylon - Modified variant of Nylon 645. Aimed at increasing print bed adherence, reducing water absorption and thermal expansion while remaining transparent for applications in non-destructive testing of the internal structures in 3D printed models.

- PCTPE - Plasticized copolyamide thermoplastic elastomer is a chemical blend of nylon with thermoplastic elastomer (TPE), aiming to produce a more user-friendly flexible model filament that maintains the positive mechanical durability of nylon.

Nylon filaments show the standard increasing absorption with frequency in the $\mathrm{THz}$ region, as can be seen in Fig. 2c. No absorption peaks are observed across the spectrum. Overall, the absorption of these filaments is considerably high. For frequencies above $0.75 \mathrm{THz}$, the absorption coefficient is $>20 \mathrm{~cm}^{-1}$ rising significantly, rendering optical devices in this region unusable. For frequencies below $0.75 \mathrm{THz}$, the absorption does steadily drop, but insufficiently to be considered a candidate for wide range applications in $\mathrm{THz}$ optical devices. Even at the lowest point, the transmissive losses per $\mathrm{cm}$ of bulk are still above $75 \%$, forcing any printed optics to have interaction lengths of a few millimeters at most.

Interestingly, the spectra are similar for all the nylon variants, despite the changes in composition, production and in the case of PCTPE, blending with another polymer. This suggests that the nylon base dominates the spectral profile. Furthermore, it can 
be concluded that TPE is relatively transmissive in the THz regime, at least in this form. Importantly, the spectral consistency across the filaments permits end users to choose a product based on their specific requirements without sacrificing optical properties.

As expected, the pure nylon-based filaments share similar refractive indices. From Fig. 3c, white nylon has a refractive index of $1.75 \pm 0.03$ across the spectrum. 645 and 910 modifications show refractive indices of $1.75 \pm 0.01$ and $1.71 \pm 0.01$, respectively, with bridge having a $1.70 \pm 0.01$ refractive index. PCTPE is somewhat lower at $1.61 \pm 0.01$ for $\mathrm{THz}$ frequencies. Unlike the absorption coefficients, the refractive indices reflect the subtle changes in the nylon filaments as well as the more substantial modification in the PCTPE filament. The refractive index for 645,910 , bridge and white nylon shows a subtle drop with increasing frequency. For visible photons, the refractive index of these nylons will fall in the range of 1.50-1.53, depending on the exact modification [40]. The $\mathrm{THz}$ data is in agreement with this, with the higher refractive index materials dropping towards this region. PCTPE, whose $\mathrm{THz}$ refractive index is much closer to the visible case, remains constant.

Overall, printing with plain nylon is very difficult, as outlined previously in this section. Testing plain white nylon confirmed this, with tests peeling off the print bed even with initial layers being glued down with an acetone-ABS slurry. However, the modified variants were found to be a marked improvement and are somewhat similar in print difficulty to ABS. Printing will require a heated print bed around $70^{\circ} \mathrm{C}$ and a hot end capable of producing extrusion temperatures in the vicinity of $250-260^{\circ} \mathrm{C}$. For extending prints into $\mathrm{THz}$ devices, the mechanical properties are suitable for the modified 645, 910, bridge and PCTPE variants. Considering 910 and bridge are improvements on 645, while being optically identical, they would be better equipped to produce $\mathrm{THz}$ optics. Despite this, when considering the required hardware, user experience and high absorbance, nylon filaments are generally unsuitable for $\mathrm{THz}$ devices across the spectrum save for small, niche applications.

\subsection{Polycarbonate}

Polycarbonate is somewhat of a niche filament for 3D printing applications. It consists of very desirable mechanical properties including superior toughness and impact resistance over all other 3D printer filaments. This is offset however, with polycarbonates thermal properties, which make it a very difficult to use. Polycarbonate has a relatively high glass transition temperature of $150^{\circ} \mathrm{C}$, which implies high extrusion temperatures in the $3 \mathrm{D}$ printing process, typically $300^{\circ} \mathrm{C}$. Furthermore, polycarbonate has a high linear thermal expansion coefficient of $70 \times 10^{-6}{ }^{\circ} \mathrm{C}^{-1}$ [48]. The coupling of these two thermal properties leads to large warping of prints in conjunction to pushing standard commercial 3D printer hardware to the limits of operation. Suppliers quote the required temperature for the print bed to be 'as high as possible' further illustrating the extreme setups required to produce successful models.

As with many other filaments, blending of polycarbonate has been attempted to reduce harsh printing conditions. Typically made with ABS, attention is required to optimize mechanical properties while sufficiently improving thermal properties. For these blends the optimal in mechanical properties is found for a polycarbonate 
concentration of $90 \%$ [49]. Experimental data has shown that outside this 90/10 ratio, most mechanical properties are weaker than both pure ABS and polycarbonate.

Experimental data in Fig. 2d shows desirable optical properties in the $\mathrm{THz}$ region for standard polycarbonate filaments. The absorption coefficient is below $10 \mathrm{~cm}^{-1}$ for frequencies below $1 \mathrm{THz}$ before further dropping below $5 \mathrm{~cm}^{-1}$ for frequencies below $0.7 \mathrm{THz}$. Thus, 3D printed quasi-optics for $\mathrm{THz}$ applications are achievable in the $<0.7 \mathrm{THz}$ range, while thin dielectric devices are still feasible up to $1 \mathrm{THz}$. Above $1 \mathrm{THz}$ absorption losses steadily increase and become too high to be suitable for $\mathrm{THz}$ applications.

The polycarbonate/ABS blended filament shows a significant increase in absorption across the spectrum. In this case, the absorption coefficient only drops below $10 \mathrm{~cm}^{-1}$ for frequencies less than $0.8 \mathrm{THz}$ and fails to fall below the $5 \mathrm{~cm}^{-1}$ threshold. As expected, with higher photon energy, the absorption increases. From the experimental data, the added ABS serves to dominate the optical spectrum, with the spectral profile consistent with standard ABS until 1.5 THz. Both the standard and blended filaments are featureless across the spectrum.

The refractive index of the standard polycarbonate is observed to be $1.49 \pm 0.03$. With ABS showing a similar refractive index of $1.49 \pm 0.01$, the blended filament gives the expected result of $1.51 \pm 0.01$, agreeing within experimental uncertainty, as can be seen in Fig. 3d. Standard polycarbonate hints at a very slight decrease in refractive index with frequency. This is emphasized by the gradual separation of the standard and ABS blended curves. This is not in agreement in comparison to the visible light refractive index of polycarbonates, given as 1.53-1.59 [40]. This suggests a local minima must be present for the polycarbonate filament between the $\mathrm{THz}$ and visible regions of the electromagnetic spectrum.

Optically, pure polycarbonate filament shows promise in 3D printing $\mathrm{THz}$ optics up to $1 \mathrm{THz}$. Unfortunately, this is diminished with the blended filaments with the ABS mix increasing the overall absorption to the same as standard ABS filament up to $1.4 \mathrm{THz}$. Thus, in this region, given that ABS is far easier to print with it would be a much better choice. Above $1.4 \mathrm{THz}$, there is a slight decrease in the blended filament curve compared to the ABS, but the overall absorption losses are so severe that this filament is not usable in this range.

The downside to using polycarbonate for $\mathrm{THz}$ applications is the extreme print conditions. High levels of user expertise are essential for successful prints with this filament. The extrusion temperature of $300^{\circ} \mathrm{C}$ is outside the capabilities of standard commercial systems and will melt the PTFE tubing between the filament drive gear and heater block. Some level of avoidance of this problem can be achieved by lowering the extrusion temperature below the ideal. Polycarbonate changes phase over a large temperature range so extrusion can be achieved as low as $275^{\circ} \mathrm{C}$. To offset the temperature drop, a slower print speed must be selected. These work against each other when thermal expansion is factored. A slow print speed allows parts more time to cool and exaggerates already significant thermal expansion. Ideally, prints need to be performed at higher temperatures and faster. Some level of warping is unavoidable with polycarbonate. This can be minimized with a very high bed temperature $>130{ }^{\circ} \mathrm{C}$ and by physically sticking prints to the build plate with an ABS slurry. However, test prints of lenses showed that after some time, the edges would still warp and pull the surface layer off the build platform. 
Overall, pure polycarbonate is a viable candidate for $\mathrm{THz}$ optical applications provided sufficient hardware and user experience are available. The mechanical properties are ideal for delicate and fine elements and a reasonable transparency window is available to take advantage of. Large thermal expansion limits the most successful prints to ones smaller in size and with high degrees of rotational symmetry. Furthermore, if the mechanical properties of the $\mathrm{THz}$ device are not so important, other filaments discussed provide comparable and better optical properties while being much easier to use, thus being more suitable for $3 \mathrm{D}$ printed $\mathrm{THz}$ equipment.

\subsection{Polypropylene}

Polypropylene is generally considered an experimental 3D printer filament. Despite this, development of a useable filament for FDM printers is desirable owing to large array of applications and consumption by society. Polypropylene is considered a tough plastic with the desirable attributes such as fatigue resistance and low friction. This makes a prime candidate for use in hinges, gears and contacting surfaces. Polypropylene also consists of decent chemical resistance seeing applications in packaging for cleaning products and other liquid containers. Polypropylene has a large thermal expansion, with a linear coefficient of $200 \times 10^{-6}{ }^{\circ} \mathrm{C}-1$. $\mathrm{A}-13^{\circ} \mathrm{C}$ glass transition temperature and $\sim 130^{\circ} \mathrm{C}$ melting point lead to extrusion temperatures in the vicinity of $230^{\circ} \mathrm{C}$. However, successful print temperatures are highly variable between setups due to the current experimental nature of the filament.

Experimentally, polypropylene filament continues the trend showing a steadily increasing absorption with frequency as shown in Fig. 2e. The spectral envelope is featureless. A useable region is observed below $0.6 \mathrm{THz}$ with the absorption coefficient dropping below $10 \mathrm{~cm}^{-1}$. This further drops below $5 \mathrm{~cm}^{-1}$ for frequencies below $0.4 \mathrm{THz}$ suggesting viability for diffractive optics in this region. Above $0.6 \mathrm{THz}$, the absorption becomes too great to print optics devices, with transmissive losses above $90 \%$ for frequencies above $0.6 \mathrm{THz}$ and $99 \%$ for frequencies above $1.15 \mathrm{THz}$.

Although, the overall absorption of polypropylene is somewhat less than many other filaments investigated in this work, it is still significantly higher than expected. In Ref. [50], the absorption coefficient of a polypropylene filament is given as $<5 \mathrm{~cm}^{-1}$ across the same spectrum as presented here. Two possibilities are attributed to this discrepancy. Firstly, a possible error in the experimental setup. For this sample, generating consistent, homogenous specimens for spectroscopic purposes was difficult. However, numerous geometric shapes and multiple measurements were performed across many samples and found to give consistent results. So it is not likely that this is the origin of the abnormally high absorption. This is further backed up by the same process generating consistent expected results for all other tested filament samples. Secondly, and likely the origin of this discrepancy, is the actual composition of the filament is not entirely polypropylene, or at least a different modification to other tested polypropylene materials. The manufacturer specifications do not give definitive data pertaining to any blending, or true composition, of the filament. It is merely sold as a proprietary homopolymer polypropylene filament. Given 
the extreme print conditions required for polypropylene, it is likely the supplier has added other material like ABS or developed a unique filament modification, to try and reduce these issues. As can be seen in Fig. $2 \mathrm{~d}$ for polycarbonate, blending with other thermoplastics can significantly alter the optical properties, and in Fig. 2f, the same is observed for filament modifications in PET.

Figure $3 \mathrm{e}$ shows a consistent refractive index of $1.48 \pm 0.01$. This is flat across the spectrum and is close the refractive index of 1.495 calculated in Ref. [50]. Interestingly, this slight change is further evidence of the previous hypothesis that this polypropylene is modified, as other tested filaments showed similar small changes in the refractive index between base and altered variants. Figure $3 a, c, f$ illustrates this phenomenon in PLA, nylon and PET blended/ altered filaments. The refractive index for polypropylene is constant for $\mathrm{THz}$ frequencies. Interestingly, this agrees with the refractive index of polypropylenes in the visible spectrum of 1.47-1.50 [40].

In terms of the printability for $\mathrm{THz}$ devices, despite viable optical properties, polypropylene is not feasible as a filament in FDM printing of $\mathrm{THz}$ components. This boils down to the thermal properties of the filament. The large expansion makes prints almost impossible. Only small fast prints with a single axis of rotational symmetry are possible, and even then they are extremely difficult. Test prints with the edges physically glued to the build platform were observed to bubble at the centre, a consequence of the extreme warping. Furthermore, any geometry of desired $\mathrm{THz}$ prints would be difficult to reproduce accurately, further adding to the case against using polypropylene filament. Other thermoplastics tested show advantages in optical and mechanical properties as well as ease in the printability process.

\subsection{Polyethylene Terephthalate}

Polyethylene terephthalate (PET) is commonly used in the manufacturing of plastic bottles. It is a transparent, fairly hard material, with its transparency changing as it is heated and cooled in the 3D printing process [51]. The wide use and visible transparency of the thermoplastic make it an attractive candidate as a $3 \mathrm{D}$ printer filament. Not just visually appealing, the optical clarity is ideal in non-destructive analysis of the internal structure of models, giving insights highly sort after in the prototyping process. The thermal properties of PET also make it an attractive printing filament, with a glass transition temperature of $75^{\circ} \mathrm{C}$ allowing print temperatures in the vicinity of $230-260^{\circ} \mathrm{C}$ [52]. With a thermal expansion coefficient of $59 \times 10^{-6}$ ${ }^{\circ} \mathrm{C}^{-1}$, lower than most printer filaments, warping is a minimal issue with this thermoplastic. PET can be modified through copolymerization and as such, like with many filaments, proprietary modifications have been made to improve the overall print properties. These include using PETG (glycol modified PET), as well as manufacturer developed filaments like Taulman T-Glase (PETT) and Colorfabb-XT. The exact composition of these filaments is not revealed by the supplier.

The absorption coefficient of three variants of PET 3D printer filament is shown in Fig. 2f. All spectra are featureless in the THz regime; however, interesting characteristics are observed between each filament. In the low frequency region below 
0.6 THz, all the filaments are similar optically. Colorfabb-XT and PETG are identical with T-Glase showing a slightly elevated absorption. Differentiation between samples increases with higher frequency. Colorfabb-XT consistently remains the lowest absorbing material and T-Glase the strongest absorber with PETG 'in the middle'. The absorption of PETG becomes comparable to T-Glase in the 0.9-1.3-THz region before dropping lower again in the high frequency spectral region.

For THz optics, PETG and Colorfabb-XT become useable for frequencies lower than $0.62 \mathrm{THz}$ reaching the threshold of $10 \mathrm{~cm}^{-1}$ for this region. In comparison, TGlase does not reach this absorption limit until frequencies lower than $0.49 \mathrm{THz}$ are present. The ideal absorption coefficient limit of $5 \mathrm{~cm}^{-1}$ is obtained for frequencies below $0.38 \mathrm{THz}$ for PETG and Colorfabb-XT and is not reached for T-Glase in the observed spectral range. Thus, optically PET filaments are viable for $\mathrm{THz}$ optics up to $0.62 \mathrm{THz}$ and suitable for quasi-optics below $0.38 \mathrm{THz}$ for PETG and Colorfabb-XT.

From Fig. 3f, each filament shows a unique refractive index, reflective of the small composition changes between each filament. The refractive index of PETG filament is observed as $1.59 \pm 0.01$ across the spectrum, with Colorfabb-XT giving $1.64 \pm 0.02$ and T-Glase, $1.53 \pm 0.01$. T-Glase remains very consistent across the observed range, with PETG hinting at a very subtle decrease with higher frequency and Colorfabb-XT giving a slightly more significant decrease. The refractive index for PET is 1.48 in the visible spectrum [40]. This agrees with the $\mathrm{THz}$ data, as the filaments with refractive indices further from visible giving a stronger decrease across the $\mathrm{THz}$ region towards 1.48 .

Overall printability of $\mathrm{THz}$ optics with PET is certainly feasible. The required hardware and user expertise required is comparable to ABS and other standard filaments. A heated bed is recommended but not necessary and should be kept below the glass transition temperature of $70^{\circ} \mathrm{C}$. This coupled with extrusion temperatures around $250^{\circ} \mathrm{C}$ is well within the capabilities of most commercially available FDM printers. The optical properties are similar to ABS and not as desirable as HIPS but the visual transparency may provide advantages in some niche applications. For example, using PET as a window for an evacuated device would allow visible inspection of the device in operation.

\subsection{High Impact Polystyrene}

High impact polystyrene (HIPS) is a widely used FDM filament support material for dual extrusion printers. HIPS has a glass transition temperature of $100^{\circ} \mathrm{C}$ and a thermal expansion coefficient of $80 \times 10^{-6}{ }^{\circ} \mathrm{C}^{-1}$. With a glass transition temperature similar to $\mathrm{ABS}$, extrusion temperatures the same as for $\mathrm{ABS}$ are permissible. Hence, using HIPS alongside ABS in dual prints is a simple transition. Furthermore, HIPS will dissolve in a limonene bath. Consequently, HIPS is an ideal candidate as a structural support and internal material for ABS prints and is used primarily for this reason. Structural prints with this material are typically not a priority owing to inferior mechanical properties when compared to other filaments like ABS, nylon 
and PET. These include low tensile and flexural strength, low elastic modulus and elongation at break.

The absorption spectrum shown in Fig. $2 \mathrm{~g}$ for HIPS gives some very encouraging results. The spectrum is featureless, increasing steadily with frequency. Pertinently, this increase is significantly slower than with all other measured filaments. A useable frequency range up to $1.3 \mathrm{THz}$ with absorption coefficient $<10 \mathrm{~cm}^{-1}$ is observed. For frequencies below $0.88 \mathrm{THz}$, the absorption coefficient drops below $5 \mathrm{~cm}^{-1}$, with diffractive optics feasible in this region. The absorption coefficient further drops below $3 \mathrm{~cm}^{-1}$ for frequencies less than $0.62 \mathrm{THz}$, corresponding to a transmitted signal above $50 \%$ for $1 \mathrm{~cm}$ bulk of material, which is a substantial result. The low frequency rise in absorption is an artefact of the experimental equipment.

From Fig. $3 \mathrm{~g}$, the refractive index of HIPS in the THz regime is given as $1.48 \pm$ 0.01 . This is observed to be highly consistent across the observed spectrum.

\subsection{Polyvinyl Alcohol}

Polyvinyl alcohol (PVA), like HIPS, typically sees applications in FDM 3D printing as a support material with dual extrusion setups. The challenge with using PVA for this purpose is higher than HIPS, with PVA having differing thermal properties to ABS, as well as large water absorption. However, with care taken in filament storage and printer setup, successful prints are achievable [53]. Thermally, PVA melts at $104{ }^{\circ} \mathrm{C}$ leading to an extrusion temperature of $190^{\circ} \mathrm{C}$. With a thermal expansion coefficient of $70 \times 10^{-5}{ }^{\circ} \mathrm{C}^{-1}$, lower than most thermoplastics tested and comparable to PLA, warping of prints is minimal with PVA filament.

PVA filament is water soluble and cost-effective, a combination which is unique to this filament, hence interest in its use as support and internal structure material. Also, due to PVA's solubility, research is active in exploiting this characteristic and has lead to investigations in areas such as pharmaceuticals, where PVA is being used to print tablets that could be designed for tailored drug delivery to patients or to engineer specific drug release profiles [54].

From Fig. 2h, a featureless absorption increasing steadily with frequency is observed. The absorption losses for PVA are significantly higher than other tested filaments across the spectrum. Even at low frequency, the absorption coefficient is above $15 \mathrm{~cm}^{-1}$, corresponding to $>97 \%$ transmissive losses for $1 \mathrm{~cm}$ of material. Even thin printed optics will fail to be feasible with $1 \mathrm{~mm}$ of bulk still seeing $>30 \%$ transmissive losses. Therefore, 3D printed devices tailored to $\mathrm{THz}$ applications are impracticable, if not impossible, with PVA filament.

Figure $3 \mathrm{~h}$ shows the refractive index for PVA filament across the THz spectrum. This is observed to be $1.79 \pm 0.03$. Close inspection of the data hints at a decrease of the refractive index with frequency. This is consistent with the visible refractive index of 1.5 for PVA.

Overall, despite the printability of PVA being quite beneficial, poor optical properties inhibit its use in 3D printing functional $\mathrm{THz}$ optics. To reach a useable threshold, optics would have to be kept to a millimeter or smaller in thickness. This is not only at the limit of the 3D printer but also beyond the capabilities of the PVA filament, 
Table 4 Summary of 3D printer filaments in the THz regime

\begin{tabular}{lll}
\hline Filament & Absorption coefficient $<10 \mathrm{~cm}^{-1}$ & Printability \\
\hline HIPS & $0.1-1.30 \mathrm{THz}$ & easy \\
PC & $0.1-1.00 \mathrm{THz}$ & hard \\
PC/ABS & $0.1-0.80 \mathrm{THz}$ & hard \\
ABS & $0.1-0.75 \mathrm{THz}$ & medium \\
PP & $0.1-0.60 \mathrm{THz}$ & hard \\
PET (+Mods.) & $0.1-0.57 \mathrm{THz}$ & medium \\
PLA & $0.1-0.50 \mathrm{THz}$ & easy \\
PLA/PHA blend & $0.1-0.50 \mathrm{THz}$ & easy \\
Nylon (+Mods.) & $0.1-0.50 \mathrm{THz}$ & medium \\
PVA & $-0.30 \mathrm{THz}$ & medium \\
\hline
\end{tabular}

being quite flexible and malleable. Across the entire spectrum, more suitable optical properties can be found from other filaments with PVA not showing any unique benefit pertaining to the $\mathrm{THz}$ regime. Even in its main commercial use as a support or internal dissolvable material, HIPS out-performs PVA. PVA can not be extruded at similar temperatures to other filaments because if it is kept above $200{ }^{\circ} \mathrm{C}$ pyrolysis can occur which will jam the print mechanism, requiring drilling-out or replacement. The lower print temperature means adhesion between PVA supports and the print material becomes problematic. Test pieces in Ref. [53] showed a 50\% success rate in printing a PVA block sandwiched between two slabs of ABS. Therefore, PVA is superseded in all useable criteria for $\mathrm{THz}$ components and thus a poor choice for $\mathrm{THz}$ applications.

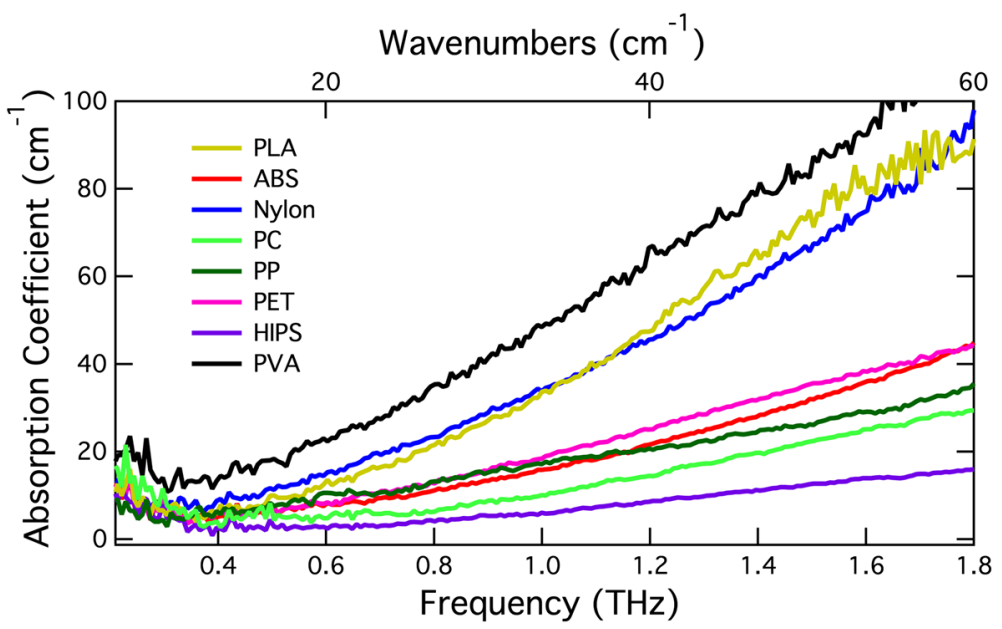

Fig. 5 Summary of absorption coefficients for 3D printer filaments in the THz regime 


\section{Conclusion}

We have evaluated the viability of standard commercially available 3D printer filaments and shown that optical properties are generally unaltered by mechanical modifications to these filaments. We conclude that ABS and HIPS filaments have the optimal compromise of a wide useable frequency range without too much complexity in achieving a successful print. The low absorption losses in HIPS are consistent with previously published values for standard polystyrene. The copolymer TOPAS, reported in literature, gives a lower absorption coefficient across the spectrum than all tested filaments in this study. However, filament of this type is not commercially available and must be developed 'in-house'. Thus, polystyrene-based filaments provide the best commercial option for $\mathrm{THz}$ printed devices, but are outperformed by TOPAS if the required infrastructure is available.

Some of the best optical products are those that are most difficult to print: PP, PC, and PC/ABS blend. Some nylon and PET modifications can fill niche applications because of their transparency in the visible spectrum. PVA printed devices are shown to be impracticable in the $\mathrm{THz}$ region. A summary of the useable frequency range and printability is shown in Table 4. A summary of the absorption coefficients is shown in Fig. 5. Here a representative from each pigment grouping is displayed for direct comparison. The increase in absorption coefficient at low frequency is an artefact of the experimental system, at the limits of the THz source.

\section{References}

1. I. Gibson, D. Rosen, and B. Stucker, Additive manufacturing technologies: $3 D$ printing, rapid prototyping and direct digital manufacturing, Springer, Berlin, 2015.

2. B. Bhushan and M. Caspers, An overview of additive manufacturing (3D printing) for microfabrication, Microsystem Technologies 23 (2017), no. 4, 1117-1124.

3. R. A. Lewis, Terahertz physics, Cambridge University Press, Cambridge, 2012.

4. C. Jördens, K. L. Chee, I. A. I. Al-Naib, I. Pupeza, S. Peik, G. Wenke, and M. Koch, Dielectric fibres for low-loss transmission of millimetre waves and its application in couplers and splitters, Journal of Infrared Millimeter, and Terahertz Waves 31 (2010), 214.

5. C. Goy, M. Scheller, B. Scherger, V. P. Wallace, and M. Koch, Terahertz waveguide prism, Optics Express 21 (2013), no. 16, 19292-19301.

6. L. Lo and R. Leonhardt, Aspheric lenses for terahertz imaging, Optics Express 16 (2008), no. 20, 15991-15998.

7. J. L. Coutaz, F. Garet, E. Bonnet, A. V. Tishchenko, O. Parriaux, and M. Nazarov, Grating diffraction effects in the THz domain, Acta Physica Polonica 107 (2005), no. 1, 26-37.

8. N. S. Stoyanov, T. Feurer, D. W. Ward, and K. A. Nelson, Integrated diffractive terahertz elements, Applied Physics Letters 82 (2003), no. 5, 674.

9. B. Zhang, Y.X. Guo, H. Zirath, and Y. P. Zhang, Investigation on 3-D-printing technologies for millimeter-wave and terahertz applications, Proceedings of the IEEE 105 (2017), no. 4, 723-736.

10. A. D. Squires, E. Constable, and R. A. Lewis, 3D printed terahertz diffraction gratings and lenses. Journal of Infrared, Millimeter, and Terahertz Waves 36 (2015), no. 1, 72-80.

11. W. D. Furlan, V. Ferrando, J. A. Monsoriu, P. Zagrajek, and E. Czerwinska, 3D printed diffractive terahertz lenses, M. Szustakowski. Optics Letters 41 (2016), no. 8, 1748-1751.

12. Z. Zhang, X. Wei, C. Liu, K. Wang, J. Liu, and Z. Yang, Rapid fabrication of terahertz lens via three-dimensional printing technology, Chinese Optics Letters 13 (2015), no. 2, 022201-022201. 
13. S. F. Busch, M. Weidenbach, J. C. Balzer, and M. Koch. 3D Printed with, THz Optics TOPAS technology. Journal of Infrared, Millimeter and Terahertz Waves 37 (2016), no. 4, 303-307.

14. J. Suszek, A. Siemion, M. S. Bieda, N. Błocki, D. Coquillat, G. Cywiński, E. Czerwińska, M. Doch, A. Kowalczyk, N. Palka, A. Sobczyk, P. Zagrajek, M. Zaremba, A. Kolodziejczyk, W. Knap, and M. Sypek, 3-D-printed flat optics for THz linear scanners, IEEE Transactions on Terahertz Science and Technology 5 (2015), no. 2, 314-316.

15. M. Weidenbach, D. Jahn, A. Rehn, S. F. Busch, F. Beltrán-Mejía, J. C. Balzer, and M. Koch, 3D printed dielectric rectangular waveguides, splitters and couplers for $120 \mathrm{GHz}$, Optics Express 24 (2016), no. 25, 28968-28976.

16. M. Liang, W. -R. Ng, K. Chang, K. Gbele, M. E. Gehm, and H. Xin, A 3-D Luneburg lens antenna fabricated by polymer jetting rapid prototyping, IEEE Transactions on Antennas and Propagation 62 (2014), no. 4, 1799-1807.

17. S. Pandey, B. Gupta, and A. Nahata, Terahertz plasmonic waveguides created via 3 D printing, Optics Express 21 (2013), no. 21, 24422-24430.

18. Z. Wu, W. -R. Ng, M. E. Gehm, and H. Xin, Terahertz electromagnetic crystal waveguide fabricated by polymer jetting rapid prototyping, Optics Express 19 (2011), no. 5, 3962-3972.

19. T. Ma, H. Guerboukha, M. Girard, A. D. Squires, and R. A. Lewis, 3D printed hollow-core terahertz optical waveguides with hyperuniform disordered dielectric reflectors, M. Skorobogatiy., Advanced Optical Materials 4 (2016), no. 12, 2085-2094.

20. W. J. Otter and S. Lucyszyn, Hybrid 3-D-printing technology for tunable THz applications, Proceedings of the IEEE 105 (2017), no. 4, 756-767.

21. D. W. Vogt and R. Leonhardt, 3D-printed broadband dielectric tube terahertz waveguide with antireflection structure, Journal of Infrared, Millimeter, and Terahertz Waves 37 (2016), no. 11, 10861095.

22. N. J. Karl, R. W. McKinney, Y. Monnai, R. Mendis, and D. M. Mittleman, Frequency-division multiplexing in the terahertz range using a leaky-wave antenna, Nature Photonics 9 (2015), 717-720.

23. N. Yudasari, J. Anthony, and R. Leonhardt, Terahertz pulse propagation in 3D-printed waveguide with metal wires component, Optics Express 22 (2014), no. 21, 26042-26054.

24. A. R. Phipps, A. J. MacLachlan, C. W. Robertson, L. Zhang, I. V. Konoplev, A. W. Cross, and A. D. R. Phelps, Electron beam excitation of coherent sub-terahertz radiation in periodic structures manufactured by 3D printing, Nuclear Instruments and Methods in Physics Research B 402 (2017), 202-205.

25. E. A. Gurvitz, S. A. Andronaki, S. I. Gusev, V. Y. Soboleva, Y. D. Nazarov, and M. K. Khodzitsky, Development of $3 D$ anisotropic artificial dielectric metamaterial for THz frequency range. PIERS Proceedings, 2715-2718, 2014.

26. B. Zhang and $\mathrm{H}$. Zirath. Metallic, 3-D printed rectangular waveguides for millimeter-wave applications, IEEE Transactions on Components, Packaging and Manufacturing Technology 6 (2016), no. 5 , 796-804.

27. A. I. Hernandez-Serrano and E. Castro-Camus, Quasi-Wollaston-prism for terahertz frequencies fabricated by $3 D$ printing, Journal of Infrared Millimeter, and Terahertz Waves 38 (2017), no. 5, 567-573.

28. H. Yi, S.-W. Qu, K.-B. Ng, and X. Bai, 3D Printed Millimeter-Wave and Terahertz Lenses with Fixed and Frequency Scanned Beam, IEEE Transactions on Antennas and Propagation 64 (2016), no. 2, $442-449$.

29. J. Li, K. Nallappan, and H. Guerboukha, M Skorobogatiy. 3D printed hollow core terahertz Bragg waveguides with defect layers for surface sensing applications, Optics Express 25 (2017), no. 4, 41264144.

30. F. Zhou, W. Cao, B. Dong, T. Reissman, W. Zhang, and C. Sun, Additive Manufacturing of a 3D Terahertz Gradient-Refractive Index Lens, Advanced Optical Materials 4 (2016), no. 7, 1034-1040.

31. N. Yudusari, D. Vogt, J. Anthony, and R. Leonhardt, Hollow core terahertz waveguide fabricated using a 3D printer. 39th International Conference on Infrared, Millimeter and Terahertz waves (IRMMW-THz), 2014.

32. D. Vogt, J. Anthony, and R. Leonhardt, Metallic and 3D-printed dielectric helical terahertz waveguides, Optics Express 23 (2015), no. 26, 33359-33369.

33. D. Headland, W. Withayachumnankul, M. Webb, H. Ebendorff-Heidepriem, A. Luiten, and D. Abbott, Analysis of 3D-printed metal for rapid-prototyped reflective terahertz optics, Optics Express 24 (2016), no. 15, 17384-17396. 
34. E. Castro-Aguirre, F. Iñiguez-Franco, H. Samsudin, X. Fang, and R. Auras, Poly(lactic acid)-mass production, processing, industrial applications, and end of life, Advanced Drug Delivery Reviews 107 (2016), 333-366.

35. K. M. Nampoothiri, N. R. Nair, and R. P. John, An overview of the recent developments in polylactide (PLA) research, Bioresource Technology 101 (2010), no. 22, 8493-8501.

36. R. M. Rasala, A. V. Janorkarc, and D. E. Hirt, Poly(lactic acid) modifications, Progress in Polymer Science 35 (2010), no. 3, 338-356.

37. F. Carrasco, P. Pagès, J. Gámez-Pérez, O. O. Santana, and M. L. Maspoch, Processing of poly(lactic acid) Characterization of chemical structure, thermal stability and mechanical properties, Polymer Degradation and Stability 95 (2010), no. 2, 116-125.

38. L.-T. Lim, R. Auras, and M. Rubino, Processing technologies for poly(lactic acid), Progress in Polymer Science 33 (2008), no. 8, 820-852.

39. I. Noda, M. M. Satkowski, A. E. Dowrey, and C. Marcott, Polymer alloys of nodax copolymers and poly(lactic acid), Macromolecular Bioscience 4 (2004), no. 3, 269-275.

40. http://polymerdatabase.com/.

41. M. Seidl, J. Safka, J. Bobek, L. Behalek, and J. Habr, Mechanical properties of products made of ABS with respect to individuality of FDM production process, Modern Machinery Science Journal 2 (2017), 1748-1751.

42. C. G. Ferro, S. Brischetto, R. Torre, and P. Maggiore, Characterization of ABS specimens produced via the $3 D$ printing technology for drone structural components, Curved and Layered Structures 3 (2016), no. 1, 172-188.

43. C.-C. Kuo, L.-C. Liu, W.-F. Teng, H.-Y. Chang, F.-M. Chien, S.-J. Liao, W.-F. Kuo, and C.-M. Chen, Preparation of starch/acrylonitrile-butadiene-styrene copolymers (ABS) biomass alloys and their feasible evaluation for 3D printing applications, Composites Part B: Engineering 86 (2016), 3639.

44. A. R. Torrado, C. M. Shemelya, J. D. English, Y. Lin, R. B. Wicker, and D. A. Roberson, Characterizing the effect of additives to ABS on the mechanical property anisotropy of specimens fabricated by material extrusion 3D printing, Additive Manufacturing 6 (2015), 16-29.

45. W.-Y. Wang, G.-H. Luo, F. Wei, and J. Luo, Electrical conductivity and thermal properties of acrylonitrile-butadiene-styrene filled with multiwall carbon nanotubes, Polymer Engineering and Science 49 (2009), no. 11, 2144-2149.

46. http://taulman3d.com/.

47. C. -C. Pai, R. -J. Jeng, S. J. Grossman, and J.-C. Huang, Effects of moisture on thermal and mechanical properties of nylon-6,6, Advances in Polymer Technology 9 (1989), no. 2, 157-163.

48. M. S. Mat-Shayuti, M. Z. Abdullah, and P. S. M. Megat-Yusoff, Thermal properties and morphology of polypropylene/polycarbonate/polypropylene-graft-maleic anhydride blends, MATEC Web of Conferences 69 (2016), 03001.

49. R. Krache and I. Debbah, Some mechanical and thermal properties of PC/ABS blends, Materials Sciences and Applications 2 (2011), no. 5, 404-410.

50. S. F. Busch, M. Weidenbach, M. Fey, F. Schäfer, T. Probst, and M. Koch, Optical properties of $3 D$ printable plastics in the $\mathrm{THz}$ regime and their application for $3 \mathrm{D}$ printed $\mathrm{THz}$ optics. Journal of Infrared, Millimeter, and Terahertz Waves 35 (2104), no. 12, 993-997.

51. S. Wojtyła, P. Klama, and T. Baran, Is 3D printing safe? analysis of the thermal treatment of thermoplastics: ABS, PLA, PET, and nylon, Journal of Occupational and Environmental Hygiene 14 (2017), no. $6,80-85$.

52. L. Trhlíková, O. Zmeskal, P. Psencik, and P. Florian, Study of the thermal properties of filaments for 3 D printing, AIP Conference Proceedings 1752 (2016), 040027.

53. C. Duran, V. Subbian, M. T. Giovanetti, J. R. Simkins, and F. R. Beyette, Experimental desktop $3 D$ printing using dual extrusion and water-soluble polyvinyl alcohol, Rapid Prototyping Journal 21 (2015), no. 5, 528-534.

54. A. Goyanes, A. B. M. Buanz, A. W. Basit, and S. Gaisford, Fused-filament 3D printing (3DP) for fabrication of tablets, International Journal of Pharmaceutics 476 (2014), 88-92. 